Materials Science Applications

Direct Visualization of the Earliest Stages of Crystallization

Singh, Manish Kumar et al.

Molybdenum in gunshot residue: experimental evidences and detection challenges in presence of lead and sulfur

Romolo, Francesco et al.

Quantitative 3D characterization of nanoporous gold nanoparticles by transmission electron microscopy

Mahr, Christoph et al.

Behavior of the $\varepsilon-\mathrm{Ga}_{2} \mathrm{O}_{3}: \mathrm{Sn}$ evaporation during laser-assisted atom probe tomography Rigutti, Lorenzo et al.

Quantification of trace-level silicon doping in $\mathrm{Al}_{\mathrm{x}} \mathrm{Ga}_{1-\mathrm{x}} \mathrm{N}$ films using wavelength-

dispersive X-ray microanalysis

Spasevski, Lucia et al.

Evaluation of dihedral angle twin boundaries in Cu10 wt\% $\mathrm{Zn}$ alloy using atomic

force microscopy

Starostina, Nataliya et al.

Software and Instrumentation

py4DSTEM: a software package for multimodal analysis of four-dimensional scanning

transmission electron microscopy datasets

Savitzky, Benjamin et al.

Scattering matrix determination in crystalline materials from $4 \mathrm{D}$ scanning

transmission electron microscopy at a single defocus value

Findlay, Scott et al.

Quantifying real-time sample temperature under the gas environment in the transmission electron microscope using a novel MEMS heater

Li, Meng et al.

Minimizing Crinkling of Soft Specimens Using Holey-Gold Films on Molybdenum

Grids for Cryogenic Electron Microscopy

Jiang, Xi et al.

An Acquisition Parameter Study for Machine-Learning-Enabled Electron

Backscattered Diffraction

Vecchio, Kenneth and Kaufmann, Kevin

Fast Grain Mapping with Sub-Nanometer Resolution Using 4D-STEM with

Grain Classification by Principal Component Analysis and Non-Negative Matrix Factorization

Allen, Frances et al.

Deep Learning Segmentation of Complex Features in Atomic-Resolution Phase

Contrast Transmission Electron Microscopy Images Sadre, Robbie et al.

Preparation of samples for large-scale automated electron microscopy of tissue and cell ultrastructure

Bachmann, Sebastian et al.

Focused ion beam sample preparation for in-situ thermal and electrical transmission electron microscopy

Radić, Dražen et al.

Biological Applications

Immunofluorescence Image Feature Analysis and Phenotype Scoring Pipeline for Distinguishing Epithelial-Mesenchymal Transition Weinberg, Seth et al.

Early upper aerodigestive tract cancer detection using electron microscopy to reveal chromatin packing alterations in buccal mucosa cells Robinson, Dominic et al.

Effects of iron oxide nanoparticles on structural organization of hepatocyte chromatin: Gray level co-occurrence matrix analysis Pantic, Igor et al.

Altered renal pathology in an autoimmune disease mouse model after induction of diabetes mellitus Ichii, Osamu et al.

Pathological and Immunohistochemical Microscopy of Natural Cases of Canine and Feline Neoplastic Mammary Lesions Khafaga, Asmaa et al.

Integrated microscopy and metabolomics to test an innovative fluid dynamic system for skin explants in vitro Calderan, Laura et al.

\title{
Micrographia
}

Histological, Histochemical and Ultrastructural Approach to the Ductus Deferens in Male Nile Monitor Lizard (Varanus niloticus)

$$
\text { Awad, Mahmoud et al. }
$$

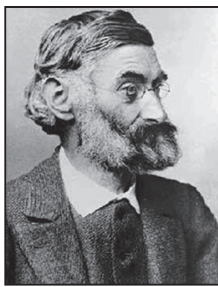

\section{Dear Abbe}

\section{Dear Abbe,}

I was recently at a demo for a new microscope and watched the rep going through the software setup. Part of the setup was creating a "user configuration." Imagine my excitement at seeing this! I would finally be able to configure my facility's users to use the microscopes correctly! Unfortunately, I found out that this really just meant configuring the software for the user. How much longer must we wait to be able to configure actual users?

\section{Disappointed in Decatur}

\section{Dear Disappointed,}

I sympathize with you. Many times I have wished to reconfigure users, usually with something heavy and blunt such as the Lab Bat. Unfortunately, with today's litigious environment, the Lab Bat is becoming a liability. Consequently, due to several lawsuits, we at Abbe Labs have been working on this very problem. You may be familiar with OLEDs, flexible transistors, and wearable electronics. And are you familiar with latex masks used in theatrical makeup? No, we aren't talking about a Lady Gaga production prop. Instead, we take a mask, and using OLEDs, flexible transistors, and other necessary bits of circuitry (all proprietary of course), - wunderbar! - we can make the user's appearance configured to whatever we desire. This, of course, does nothing for how they operate the microscope, but at least we can have our very own Phantom of the Microscope, or a famous scientist taking instruction from us when the Dean brings a tour of big donors through the facility. If nothing else, the resulting pics look great on our MySpacebook ${ }^{\mathrm{PB}}$ social media page.

Don't be caught empty-handed when the chips are down! Ante up with confidence as you deal out some advice provided by Herr Abbe. Contact him through his questionably competent sidekickat johnshields@gmail.com.

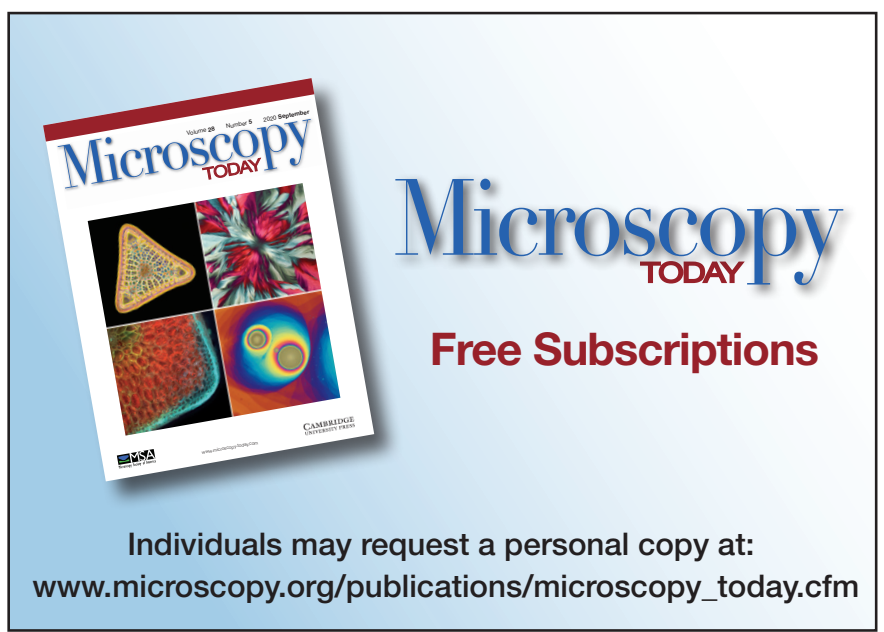




\section{Microscopy of America Awards

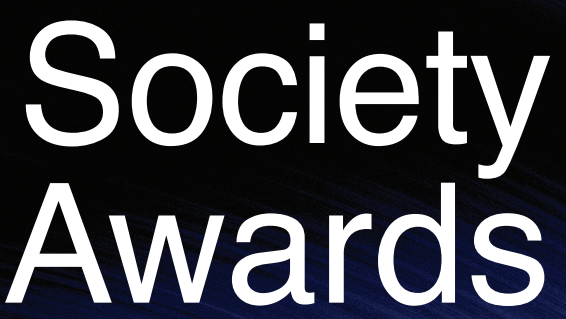

Nominations will open in August for the Microscopy Society of America Annual Awards. The awards process is one way in which the Microscopy Society of America recognizes the significant and diverse contributions that individuals make to our field. Deserving nominations for consideration should be submitted electronically no later than October 31st, 2021 to:

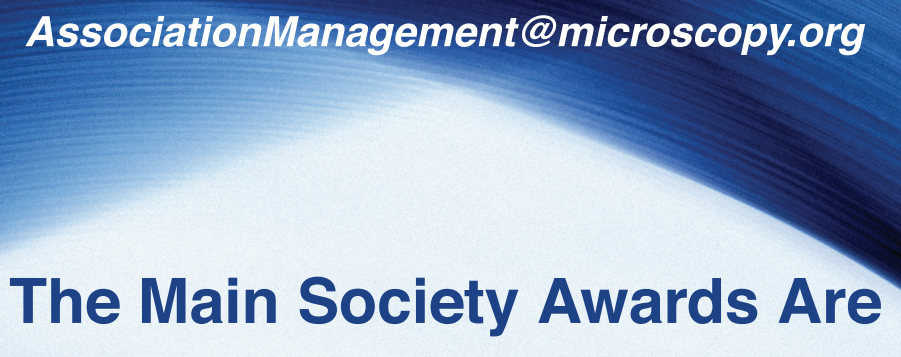

\section{Distinguished Scientist Awards}

These Awards recognize preeminent senior scientists from both the Biological and Physical disciplines who have a long-standing record of achievement during their career in the field of microscopy or microanalysis.

\section{Burton Medal}

The Burton Medal was initiated to honor the distinguished contributions to the field of microscopy and microanalysis of a scientist who is less than 40 years of age on January 1 st of the award year. (Please note the change in the selection criterion regarding age.)

\section{Outstanding Technologist Awards}

These Awards honor technologists from both the Biological (Hildegard $\mathrm{H}$. Crowley Award) and Physical Sciences (Chuck Fiori Award) who have made significant contributions such as the development of new techniques which have

contributed to the advancement of microscopy and microanalysis.

\section{Morton D. Maser Distinguished Service Award}

This Award was initiated to recognize outstanding volunteer service to the Society as exemplified by Mort Maser, who served the Society for many years with great dedication. This award is made to honor an MSA member who has provided significant volunteer service to the Society over a period of years.

\section{The Albert Crewe Award}

The Albert Crewe Award was initiated to recognize the distinguished contributions to the field of microscopy and microanalysis in the physical sciences of a postdoctoral fellow of not more than 6 years' standing (since doctoral graduation).

\section{The George Palade Award}

The George Palade Award was initiated to recognize the distinguished contributions to the field of microscopy and microanalysis in the life sciences of a postdoctoral fellow of not more than 6 years' standing (since doctoral graduation).
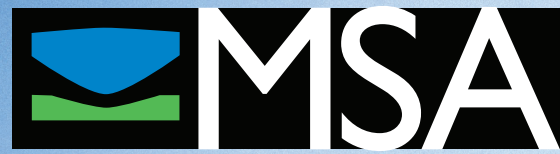

Microscopy Society of America
Further details of the nomination process can be found on the society webpage at: www.microscopy.org 